\title{
Wireless Bioradar Sensor Networks for Speech Detection and Communication
}

\author{
Ying Tian, Sheng Li, Jianqi Wang* \\ College of Biomedical Engineering, The Fourth Military Medical University, Xi'an, China \\ Email: ${ }^{*}$ cyt_bora@sina.com
}

Received 2013

\begin{abstract}
Wireless multimedia sensor networks (WMSN) are emerging to serve for the collection of acoustic and image information. In the WMSN, the microphone is usually employed to function as sensor nodes for the acquisition of acoustic data. However, those microphone sensors are needed to be placed close with sound source and cannot detect sound signal through certain obstacles. To overcome the shortcomings of microphone sensor, we develop a new type of bioradar sensor to achieve non-contact speech detection and investigate theoretically the mechanism of bioradar for speech detection. Results show that the system can successfully detect speech at some distance and even through non-metallic objects with certain thickness. In addition, in order to suppress the noise and improve the quality of the detected speech, we use spectral subtraction and Wiener filtering algorithm respectively to enhance the bioradar speech and evaluate the performance of the two methods using spectrogram.
\end{abstract}

Keywords: Bioradar Sensor; Speech Detection; Wireless Sensor Networks

\section{Introduction}

The availability of low-cost acoustic and image sensors allows for the emergence of wireless multimedia sensor networks (WMSN) [1], which can sense and transmit not only scalar sensor data but also still images, video and audio streams through multihop wireless links between sensors [2]. The WMSN usually uses the microphone to retrieve audio information. However, those microphone sensors are needed to be placed close with sound source and cannot detect sound signal through certain obstacles. Since the deployment environment is complex and diverse for various WMSN applications, the obstacles in the open area and the detection distance are the critical factors for consideration when detecting sound signal. The mechanism of microphone sensor limits its application in the area of human speech detection for those special environments. Therefore we propose to employ bioradar sensor for speech detection.

The bioradar, which can emit electromagnetic wave and extract information from echo signal in a noninvasive, safe, fast, portable fashion, has raised the interest of research in its medical application such as heart beat and respiration monitoring, and early stage breast cancer detection [3-5]. Among its promising medical applications, some reports on speech related topics further confirm the feasibility of using bioradar as sensor nodes for speech

${ }^{*}$ Corresponding author. detection. For example, Holzrichter et al. use very low power electromagnetic (EM) wave sensors to measure speech articulator motions as speech is produced [6]. $\mathrm{Li}$ discovers that using $40 \mathrm{GHz}$ dielectric integrated radar can detect and identify out exactly the existential speech signals in free space from a person speaking [7].

In this paper, we investigate theoretically the mechanism of bioradar for speech detection and develop a 35.5 $\mathrm{GHz}$ microwave bioradar which can detect speech at some distance by emitting radio waves in the microwave range to a target subject and extracting speech information from echo signal. In addition, in order to suppress the noise and improve the quality of the detected speech, we use spectral subtraction, and Wiener filtering algorithm respectively to enhance the bioradar speech and evaluate the performance of the two methods using spectrogram.

\section{System Architecture}

As shown in Figure 1, the wireless bioradar sensor network is a pivotal part of the multi-tier speech detection system. The lowest tier includes a number of bioradar sensors and relay sensors. The resources in those sensors are generally very limited in terms of power, memory and computational capability. Hence the bioradar sensor mainly deals with detection of speech and some simple processing. Due to the output of the bioradar front end is 
analog signal along with noises and very weak, we design a preprocessing circuit board to improve the signal-to-noise ratio and convert the analog signal to digital signal. The communication capability of the bioradar sensor is very limited, and it cannot communicate with gateway by a single-hop mode. Hence we design relay sensors to transmit the data collected from the sensor node by a multi-hop mode. The relay sensor only forwards packets. Advanced processing is left to the upper tier.

The second tier is gateway. It provides a transparent interface to the wireless bioradar sensor network and an interface to central server via Internet. Depending on the end-user application, we can employ corresponding device, such as CDMA gateway, WLAN gateway or GPRS gateway. The capability of gateway in computation, storage, and communication is greater than the bioradar sensor and relay sensor. It is responsible for a number of tasks, including sensor registration, initialization, customization, time synchronization, data retrieval and processing, and data fusion [8].

The third tier is central server where the large amount of data collected can be interpreted and analyzed. It can implement algorithms to identify an unexpected situation from the speech information and trigger required actions. In addition, the central server provides a graphical interface for real-time monitoring and also an interface for the definition and configuration of the system's overall behavior [9].

\section{Theoretical Analysis}

As a mechanical effect, sound is essentially the passage of pressure fluctuations through an elastic medium as the result of vibrational forces acting on that medium [10]. Speech as a type of sound source not only possesses the physical attributes as other sounds do, but also has unique attributes related with physiology, since it originates from the motion of human vocal apparatus. As shown from the mid-sagittal plane of vocal tract in Figure 2, speech is the acoustic product of the coordinated operation of lung, trachea, larynx, pharynx, nose and mouth. With the contraction of the rib cage and increase of lung pressure, air is forced from the lungs to pass through the trachea and get to larynx, where it causes the elastic vocal folds to vibrate and produces a quasi-periodic train of air pulses to excite the acoustic system. This pulse train is modified by the resonances of the vocal tract according to its change in cross-sectional area due to the movement of the articulators (e.g., the lips, jaw, tongue and soft palate, producing distinguishable voice sounds [11]. This vibration information of sound can be detected by bioradar due to the doppler effect [12].

If $S_{R}(t)$ is an ideal transmitted signal of continuous waveforms $(\mathrm{CW})$ radar, then

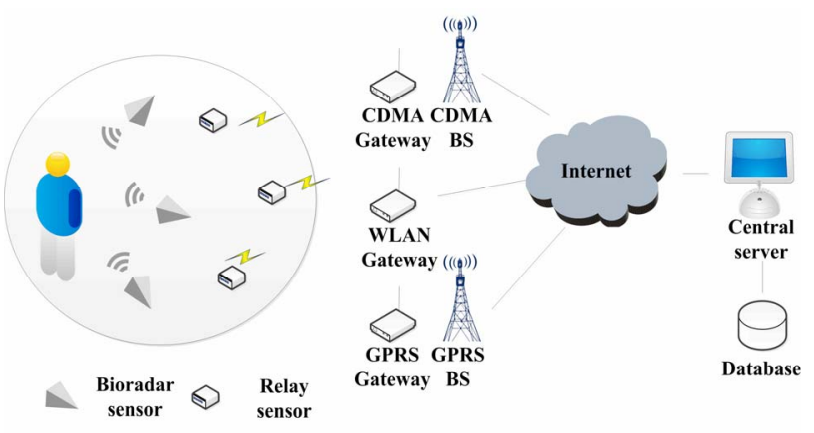

Figure 1. Speech detection system architecture.

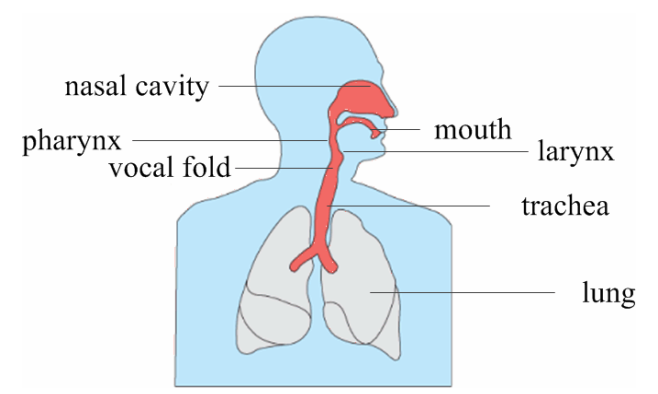

Figure 2. Diagram of mid-sagittal plane of vocal tract.

$$
S_{R}(t)=A \sin \left(2 \pi f_{0} t+\theta_{0}\right)
$$

where $A$ is the amplitude of the transmitted signal, $f_{0}$ is the frequency of the transmitted signal and $\theta_{0}$ is the initial phase. Because of the production of speech causing vibration and in turn causing the doppler effect on the received signal, its phase is changed. We denote the change of phase by $\theta(t)$, and thus the received signal can be written by

$$
\begin{aligned}
S_{\tau}(t) & =K_{1} S_{R}(t-\tau) \\
& =K_{1} A \sin \left[2 \pi f_{0}(t-\tau)+\theta_{0}+\theta(t)\right]
\end{aligned}
$$

where $K_{1}=$ attenuation coefficient and $\tau=2 R / c . R$ is the target distance and $c$ is the velocity of light. In the mixter, the transmitted signal is mixed with the received signal, and we get the mixed signal

$$
\begin{aligned}
S_{F}(t)= & A \sin \left(2 \pi f_{0} t+\theta_{0}\right) \\
& \cdot K_{1} A \sin \left[2 \pi f_{0}(t-\tau)+\theta_{0}+\theta(t)\right]
\end{aligned}
$$

After filtering out the high-frequency and DC component, we obtain

$$
S_{F}(t)=K \sin \theta(t)
$$

where $K$ is the gain of mixing and filtering. Because the change of phase is small and $\sin \theta(t) \approx \theta(t)$ for small $\theta(t)$, equation (4) can be more simply written as

$$
S_{F}(t) \approx K \theta(t)
$$

The varying displacement of vibration, $\chi(t)$ is linearly proportional to the varying phase of the echo signal: 


$$
\theta(t)=\frac{4 \pi}{\lambda} \chi(t)
$$

where $\lambda$ is the wavelength of bioradar signal. Substituting Eq. (6) into Eq. (5), we obtain

$$
S_{F}(t) \approx K \frac{4 \pi}{\lambda} \chi(t)
$$

This theoretical analysis suggests that the echo signal can be modulated by the vibration originating from the production of speech, thereby causing the change of its phase. This varying phase forms the base of the extraction of life parameter related with speech.

\section{Bioradar Sensor Hardware Design}

Based on the modular design theory, the bioradar sensor can be separated into three modules according to their specific functions, as shown in Figure 3. The function of sensor module is speech data acquisition; process unit plays the role of adjusting the signal to the back end application; radio module mainly deals with the transmission of speech data wirelessly. The details for each module design are given in the following section.

\subsection{Sensor Module}

The design of the bioradar front end is shown in Figure 4. In this bioradar front end, a superheterodyne receiver is employed. The advantage of using such receiver is that it employs two-step indirect-conversion transceiver, so that to mitigate the severe DC offset problem and the associated $1 / \mathrm{f}$ noise at baseband, that occurs normally in the direct-conversion receivers. The transmitting and receiv- ing antennas are both parabolic antennas with a diameter of $300 \mathrm{~mm}$, and the estimated beam width is $9^{\circ}$. The voltage controlled oscillator generates a very stable MMW at $34.5 \mathrm{GHz}$ with an output power of $100 \mathrm{~mW}$ [13].

\subsection{Process Unit}

The output speech signal of the bioradar front end has high frequency and relatively low amplitude. In order to suppress noises improving signal-to-noise ratio and to convert the analog output to digital signal, we design a preprocessing circuit as shown in Figure 5.

Preamplifier performs impedance matching between the output of bioradar front end and the input of the next circuit. It provides protection and insulation for the circuit. To meet the design requirements on stopband attenuation, we employ higher order filtering. Finally, to eliminate $50 \mathrm{~Hz}$ power-line interference, a $50 \mathrm{~Hz}$ notch filter is designed.

In addition, to improve the detection precision when performing $\mathrm{AD}$ conversion, we do not employ the built-in 14-bit ADC of CC2430, but rather choose an A/D chip, AD7792 with higher precision. The AD7792 is a low power, complete analog front end. It contains a low noise 16-bit $\Sigma$ - $\Delta$ ADC with three differential analog inputs [14].

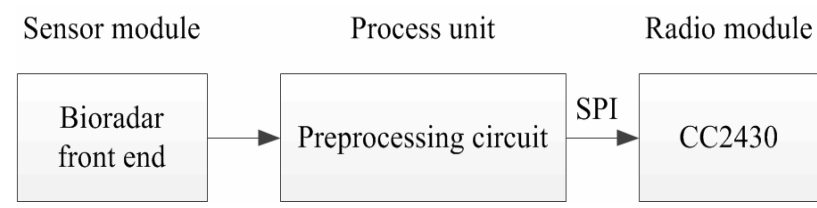

Figure 3. Block diagram of the bioradar sensor.

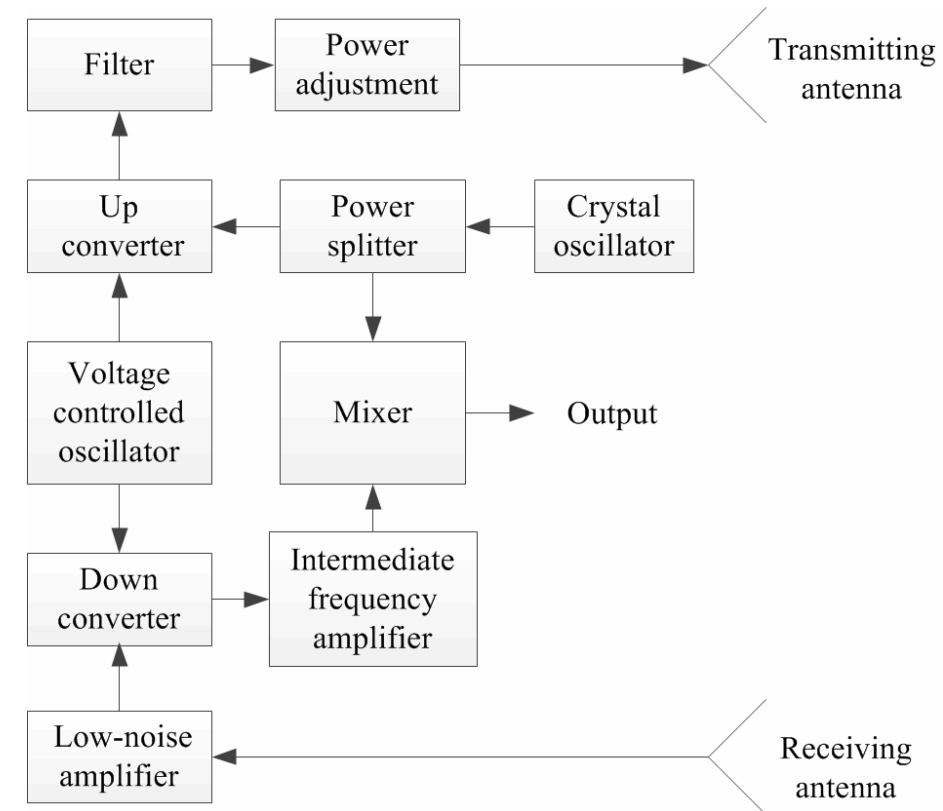

Figure 4. The schematic diagram of the bioradar front end. 


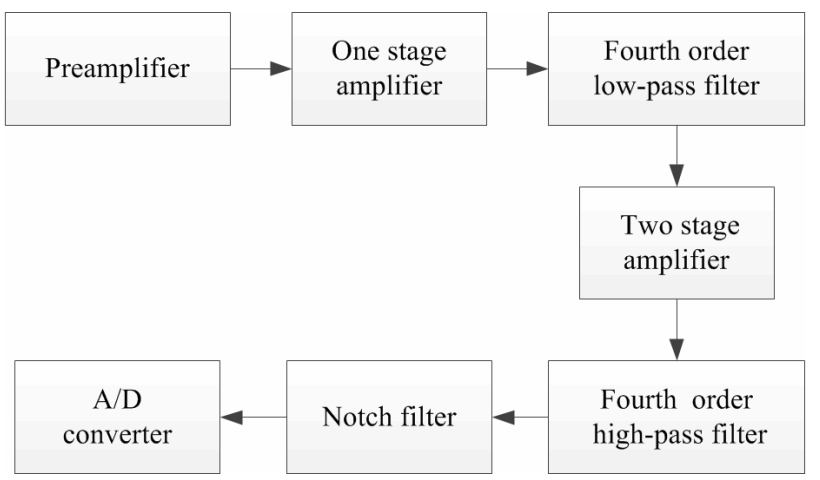

Figure 5. Block diagram of preprocessing circuit.

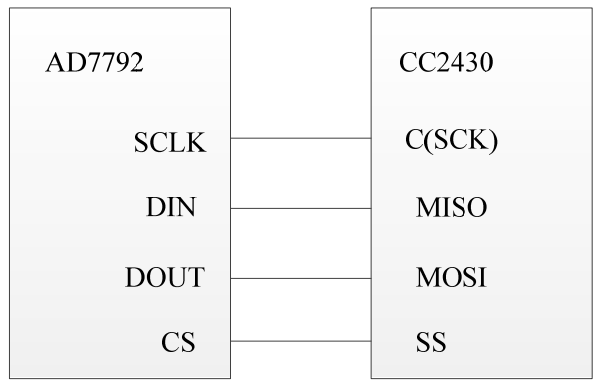

Figure 6. Interface of AD7792 and CC2430.

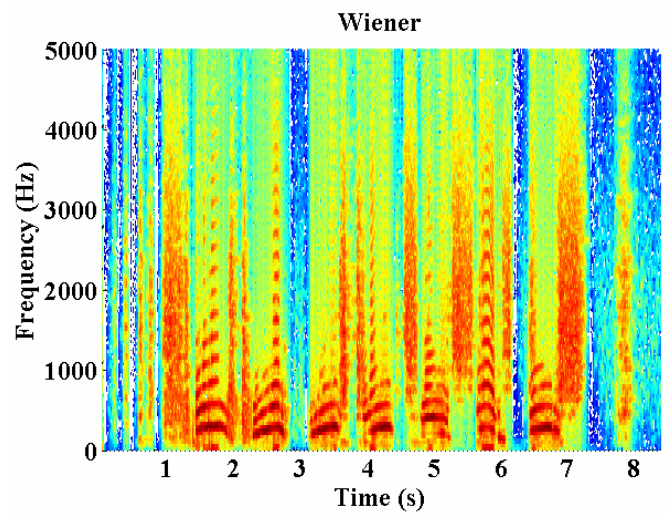

(b)

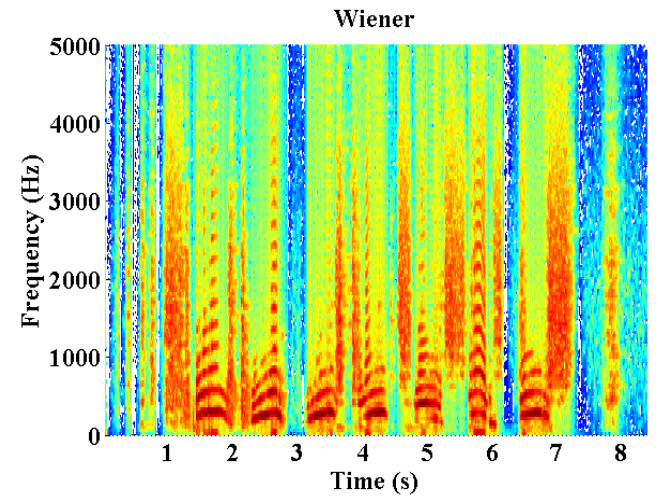

(b)

Figure 7. Spectrogram of bioradar speech. (a) speech processed by spectral subtraction. (b) speech processed by Wiener filtering.

\subsection{Radio Module}

Energy consumption is one of the major concerns when designing the sensor node. To reduce the power consumption and cost of sensor node, we choose CC2430 chip. The CC2430 is the first System-on-Chip (SoC) solution for ZigBee and it combines the excellent performance 2.4 GHz DSSS RF transceiver core with an industry-standard enhanced $8051 \mathrm{MCU}$. The CC2430 is highly suited for systems where ultra low power consumption is required. This is ensured by various operating modes. Short transition times between operating modes further ensure low power consumption [15].

Here CC2430 communicates with AD7792 using SPI (Serial Peripheral Interface) mode as shown in Figure 6.

\section{Bioradar Speech Denoising}

In our experiments, the bioradar sensor was positioned in front of the speaker and he was asked to speak seven sentences, which were recorded in a computer. The area of the room for the experiment is 172.5 square meters.

Result showed the bioradar speech was disturbed by noise. To reduce the noise and improve the quality of bioradar speech, we employed spectral subtraction and Wiener filtering algorithm respectively to enhance the detected speech. To evaluate the performance of the two methods, we plot spectrogram using Matlab. The result is shown in Figure 7. It is observed that at the nonspeech segments Wiener filtering is superior to spectral subtraction in denoising, whereas at the speech segments although Wiener filtering is still more effective in suppression of noise than spectral subtraction, it also removes some frequency components thereby causing the loss of speech information. This suggests that neither Wiener filtering nor spectral subtraction is optimal for the reduction of noise of bioradar speech, and therefore we need to explore another algorithm to suppress the noise while preserve the speech information as complete as possible not distorting the speech signal.

\section{Conclusions}

Microphone-based audio stream collection in WMSN cannot meet the requirement of speech detection in some environment. Therefore we propose to use bioradar sensor as a new method to establish wireless bioradar sensor network for speech detection. The penetrability of bioradar allows it to detect speech at some distance and even through non-metallic objects with certain thickness. The new bioradar network extends the WMSN application as well as providing us with richer information.

\section{Acknowledgements}

The work was supported by National Key Technology 
Research and Development Program of the Ministry of Science and Technology of China (No. 2012BAI20B02).

\section{REFERENCES}

[1] M. Guerrero-Zapata, R. Zilan, J. M. Barceló-Ordinas, K. Bicakci and B. Tavli, "The Future of Security in Wireless Multimedia Sensor Networks," Telecommunication Systems, Vol. 45, No. 1, 2010, pp. 77-91. doi:10.1007/s11235-009-9235-0

[2] L. You and C. G. Liu, "Robust Cross-layer Design of Wireless Multimedia Sensor Networks with Correlation and Uncertainty," Journal of Networks, Vol. 6, No. 7, 2011, pp. 1009-1016. doi:10.4304/jnw.6.7.1009-1016

[3] J. Q. Wang, C. X. Zheng, G. H. Lu and X. J. Jing, "A New Method for Identifying the Life Parameters via Radar," EURASIP Journal on Applied Signal Processing, Vol. 2007, No. 1, 2007, pp. 16-16. doi:10.1155/2007/31415

[4] H. Lv, G. H. Lu, X. J. Jing and J. Q. Wang, "A New Ultra-Wideband Radar for Detecting Survivors Buried under Earthquake Rubbles," Microwave and Optical Technology Letters, Vol. 52, No. 11, 2010, pp. 2621-2624. doi:10.1002/mop.25539

[5] S. K. Davis, B. D. Van Veen, S. C. Hagness and F. Kelcz, "Breast Tumor Characterization Based on Ultrawideband Microwave Backscatter," Biomedical Engineering, IEEE Transactions on, Vol. 55, No. 1, 2008, pp. 237-246. doi:10.1109/TBME.2007.900564

[6] J. Holzrichter, G. Burnett, L. Ng and W. Lea, "Speech Articulator Measurements Using Low Power EM-wave Sensors," Journal of the Acoustical Society of America, Vol. 103, 1998, pp. 622-625.doi:10.1121/1.421133
[7] Z. W. Li, "Millimeter Wave Radar for Detecting the Speech Signal Applications," International Journal of Infrared and Millimeter Waves, Vol. 17, No. 12, 1996, pp. 2175-2183. doi:10.1007/BF02069493

[8] A. Milenković, C. Otto and E. Jovanov, "Wireless Sensor Networks for Personal Health Monitoring: Issues and an Implementation," Computer Communications, Vol. 29, No. 13, 2006, pp. 2521-2533. doi:10.1016/i.comcom.2006.02.011

[9] H. Alemdar and C. Ersoy, "Wireless Sensor Networks for Healthcare: A Survey," Computer Networks, Vol. 54, No. 15, 2010, pp. 2688-2710. doi:10.1016/j.comnet.2010.05.003

[10] D. R. Raichel, "The Science and Applications of Acoustics," Springer Science+Business Media, New York, 2006.

[11] J. L. Flanagan, J. B. Allen and M. A. Hasegawa-Johnson, "Speech Analysis Synthesis and Perception," Springer, 2008.

[12] M. I. Skolnik, "Introduction to Radar System," McGrawHill, 1980.

[13] S. Li, Y. Tian, G. Lu, Y. Zhang, H. J. Xue, J. Q. Wang and X. J. Jing, "A New Kind of Non-Acoustic Speech Acquisition Method Based on Millimeter Wave Radar," Progress In Electromagnetics Research, Vol. 130, 2012, pp. $17-40$.

[14] AD7792 datasheet, Preliminary Technical Data AD7792/AD7793, Analog Devices, Inc.

[15] CC2430 datasheet, A True System-on-Chip solution for 2.4 GHz IEEE 802.15.4 / ZigBee ${ }^{\mathrm{TM}}$, Texas Instruments. 